\title{
The Exact Solutions for a Point Mass Moving along a Stretched String on a Winkler Foundation
}

\author{
Q. Gao, J. Zhang, H. W. Zhang, and W. X. Zhong \\ State Key Laboratory of Structural Analysis of Industrial Equipment, Department of Engineering Mechanics, \\ Faculty of Vehicle Engineering and Mechanics, Dalian University of Technology, Dalian 116023, China
}

Correspondence should be addressed to H. W. Zhang; zhanghw@dlut.edu.cn

Received 5 December 2013; Revised 13 April 2014; Accepted 10 May 2014; Published 30 June 2014

Academic Editor: Dumitru I. Caruntu

Copyright (C) 2014 Q. Gao et al. This is an open access article distributed under the Creative Commons Attribution License, which permits unrestricted use, distribution, and reproduction in any medium, provided the original work is properly cited.

This paper derives the exact solutions for a point mass moving along a stretched infinite string on a Winkler foundation at a constant velocity. The solutions for the contact force between the string and the mass are derived and then the displacement responses of the string can be obtained easily. The solutions cover infinite string subjected to a moving mass at subsonic, sonic, or supersonic velocities. When time tends to infinity, the asymptotical solutions for the contact force between the mass and the string and for the displacement of the contact point are derived. The formulas derived are shown to be correct by comparison with the semianalytical method.

\section{Introduction}

The dynamic response of a structure due to moving forces or masses is of importance in engineering applications, such as the vehicle and track interaction, the train and bridge interaction, and the pantograph and catenary interaction [1$3]$. For such problems, a lot of analytical and numerical research has been achieved.

By using the Laplace transformation, an analytical solution for a stretched infinite string subjected to a moving force at a constant speed was derived by Kanninen and Florence [4], and a set of three solutions was obtained for subsonic, sonic, and supersonic velocities. A stretched infinite string excited by a moving force with varying speed was investigated by Flaherty Jr. [5]. Using the Laplace transformation and Fourier transformation, a stretched semi-infinite string subjected to a moving force with a constant acceleration was considered by Sagartz and Forrestal [6]. Using the Laplace transformation, the dynamic response of an inhomogeneous stretched infinite string on an elastic foundation subjected to a moving force at a constant speed was investigated by Wolfert et al. [7]. An integral equation of the response of a stretched infinite string on an elastic foundation subjected to a moving force was obtained by Gavrilov [8], and an asymptotical solution was also presented. Applying the Fourier transformation, the dynamic response of a stretched infinite string on an elastically supported membrane subjected to a moving force was investigated by Dieterman and Kononov [9]. The dynamic response of two parallel stretched infinite strings on an elastically supported membrane subjected to two moving forces was obtained by Kononov and Dieterman [10]. A closed-form solution for the dynamic response of a stretched finite double-string which is connected by a Winkler elastic layer subjected to a moving harmonic force at a constant speed was presented by Rusin et al. [11].

An analytical solution for a stretched finite string subjected to a mass moving at a constant speed was presented by Smith [12], but the mass of the string was neglected in this model. A numerical solution for a stretched semi-infinite string subjected to a constantly accelerating moving mass was derived by Rodeman et al. [13], and an asymptotical solution was also obtained when the effect of the inertia of the moving mass was small. An integral equation for the response of a stretched finite string subjected to a moving mass at a constant speed was obtained by Yang et al. [14], and then they used a numerical integration method to solve this equation.

A space-time method for numerical solution of a stretched finite string subjected to a moving mass at a constant speed was proposed by Bajer and Dyniewicz [15], and then by using a combination of the Fourier integral 


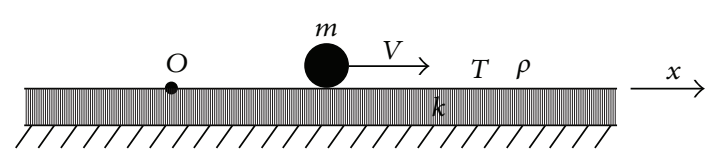

FIGURE 1: A point mass moving along a stretched string on a Winkler foundation.

transformation and time integration methods, a semianalytical method for the same problem was proposed by Dyniewicz and Bajer [16]. A moving coordinate system was introduced by Kruse et al. [17] to obtain the eigenfrequencies of a stretched infinite string on a viscoelastic foundation subjected to a two-mass oscillator at a constant speed. The Lagrange multiplier method was adopted by Lee et al. [18] to describe the dynamic responses of a coupled moving mass-stretched beam with a separation between each mass. A stretched infinite string on an elastic foundation subjected to a constant accelerating moving mass was considered by Gavrilov and Indeitsev [19], and an integral equation to describe the interaction force between the mass and the string was given and solved numerically. Many numerical methods and semianalytical solutions to solve the problems in the vibrations of structures subjected to moving loads or masses were presented by Bajer and Dyniewicz [3].

A stretched string on a Winkler foundation subjected to a moving point mass is a typical and important model to simulate the dynamic interaction between a structure and a moving mass. The exact solutions can be used as models for physical experiments and as benchmarks for testing numerical methods, and so forth. However, to the authors' knowledge, there is no exact solution for the problem of a stretched string on a Winkler foundation subjected to a moving point mass. In this paper, the exact solution for a stretched infinite string on a Winkler foundation subjected to a moving point mass is considered. The solutions cover the situation of the mass moving at subsonic, sonic, or supersonic velocities. The asymptotical solutions for the contact force between the mass and the string and for the displacement of the contact point are derived.

\section{The Governing Equation for the Moving Mass System}

As shown in Figure 1, let us consider a point mass moving along an infinite string on a Winkler foundation. The line mass density and tensile force of the string are $\rho$ and $T$, respectively, the coefficient of elasticity of the foundation is $k$, and the mass of the moving mass is $m$. The mass moves in the positive $x$ direction starting from the original point $O$ at a constant speed $V$.

Assume that the contact force between the mass and the string is denoted by $p(t)$, and then the interaction between the mass and the string can be described as in Figure 2. Therefore, the motion equation of the mass is given by

$$
m \ddot{y}=-p(t)-m g, \quad y(0)=\dot{y}(0)=p(0)=0,
$$

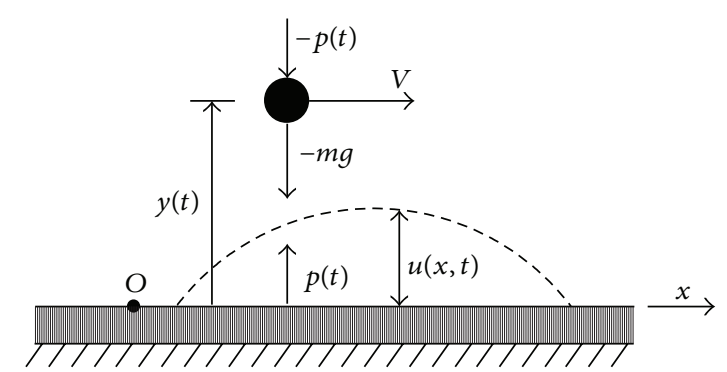

FIgURE 2: The interaction between the mass and the string.

where $y$ is the displacement of the mass and $g$ denotes the acceleration due to gravity. The motion equation and the initial conditions of the string are

$$
\begin{aligned}
\rho \frac{\partial^{2} u}{\partial t^{2}} & =T \frac{\partial^{2} u}{\partial x^{2}}-k u+p(t) \delta(x-V t), \\
u(x, 0) & =\frac{\partial u}{\partial t}(x, 0)=0,
\end{aligned}
$$

where $\delta$ denotes the Dirac delta function. The continuous condition for the mass and the string is

$$
y(t)=u(V t, t) .
$$

Therefore, (1) and (4) yield

$$
m \frac{\mathrm{d}^{2} u(V t, t)}{\mathrm{d} t^{2}}=-p(t)-m g, \quad p(0)=0 .
$$

Then, combining (2) with (5) gives the governing equation for the string subjected to a mass moving at a constant speed

$$
\begin{aligned}
\rho \frac{\partial^{2} u}{\partial t^{2}}= & T \frac{\partial^{2} u}{\partial x^{2}}-k u-m g \delta(x-V t) \\
& -m\left[\frac{\partial^{2} u(x, t)}{\partial^{2} t}+2 V \frac{\partial^{2} u(x, t)}{\partial x \partial t}+V^{2} \frac{\partial^{2} u(x, t)}{\partial x^{2}}\right]_{x=V t}
\end{aligned}
$$

in which the terms $2 m V \partial^{2} u(x, t) /\left.\partial x \partial t\right|_{x=V t}$ and $m V^{2} \partial^{2} u(x, t) /\left.\partial x^{2}\right|_{x=V t}$ are the Coriolis force and centripetal force, respectively.

Equation (2) can also be written as

$$
\frac{\partial^{2} u}{\partial t^{2}}=c^{2} \frac{\partial^{2} u}{\partial x^{2}}-\kappa^{2} u+\rho^{-1} p(t) \delta(x-V t),
$$

where

$$
c=\sqrt{\frac{T}{\rho}}, \quad \kappa=\sqrt{\frac{k}{\rho}}
$$

in which $c$ is the velocity of the wave. For different engineering applications, the value of $c$ can be different. For example, if a track is considered, the magnitude of $c$ is about $10^{3} \mathrm{~m} / \mathrm{s}$, while if the contact wire of the pantograph-catenary system is 
considered, the magnitude of $c$ is about $10^{2} \mathrm{~m} / \mathrm{s}$. The solution for (2) and (3) can be given by [20, Section 7.4]

$$
\begin{aligned}
& u(x, t) \\
& =\frac{1}{2 \rho c} \int_{0}^{t} p(\tau) \mathrm{d} \tau \int_{x-c(t-\tau)}^{x+c(t-\tau)} \delta(\sigma-V \tau) \\
& \quad \times J_{0}\left[\alpha \sqrt{c^{2}(t-\tau)^{2}-(x-\sigma)^{2}}\right] \mathrm{d} \sigma,
\end{aligned}
$$

where $J_{0}$ denotes the Bessel function of the first kind of order 0 , and

$$
\alpha=\frac{\kappa}{c} .
$$

When $V<c$, (9) can be written as

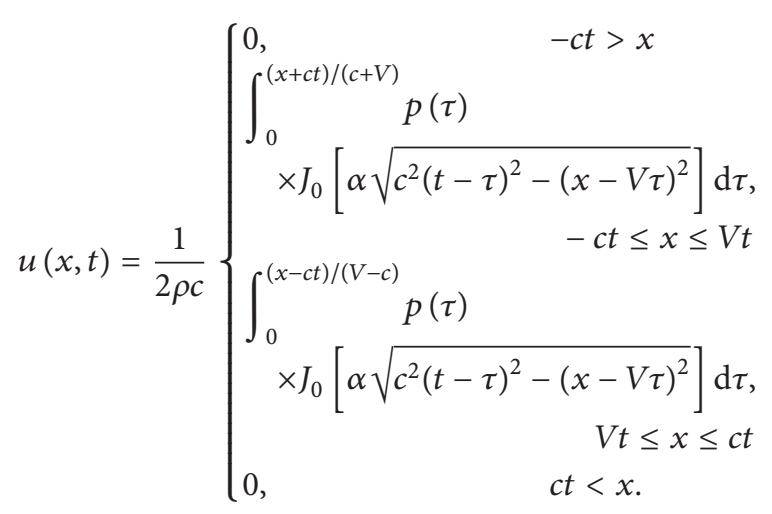

When $V>c$, (9) can be written as

$$
u(x, t)=\frac{1}{2 \rho c}\left\{\begin{array}{lr}
0, & -c t>x \\
\int_{0}^{(x+c t) /(c+V)} & p(\tau) \\
\quad \times J_{0}\left[\alpha \sqrt{c^{2}(t-\tau)^{2}-(x-V \tau)^{2}}\right] \mathrm{d} \tau, \\
\int_{(x-c t) /(V-c)}^{(x+c t) /(c+V)} \quad-c t \leq x \leq c t \\
\times J_{0}\left[\alpha \sqrt{c^{2}(t-\tau)^{2}-(x-V \tau)^{2}}\right] \mathrm{d} \tau, \\
0, \\
c t \leq x \leq V t
\end{array}\right.
$$

and when $V=c$, (9) can be written as

$$
u(x, t)=\frac{1}{2 \rho c}\left\{\begin{array}{lc}
0, & -c t>x \\
\int_{0}^{(x+c t) / 2 c} & p(\tau) \\
x J_{0}\left[\alpha \sqrt{c^{2}(t-\tau)^{2}-(x-c \tau)^{2}}\right] \mathrm{d} \tau, \\
0, & -c t \leq x \leq c t
\end{array}\right.
$$

Combining (5) with (11)-(13) gives the governing equation of the contact force when the mass moves at the subsonic, supersonic, or sonic velocities, respectively. In the following sections, the analytical solution for the contact force is derived.

\section{The Solutions When the Mass Moves at Subsonic Velocity}

When $V<c$, using (11) the following equation can be obtained as

$$
\begin{aligned}
\frac{\mathrm{d}^{2} u(V t, t)}{\mathrm{d} t^{2}} & \\
=\frac{1}{2 \rho c}\left[\dot{p}(t)+\beta \int_{0}^{t}\left\{-\beta J_{0}[\beta(t-\tau)]\right.\right. & \left.\left.+\frac{J_{1}[\beta(t-\tau)]}{t-\tau}\right\} p(\tau) \mathrm{d} \tau\right],
\end{aligned}
$$

where $J_{1}$ denotes the Bessel function of the first kind of order 1 , and

$$
\beta=\alpha \sqrt{c^{2}-V^{2}} .
$$

Then, according to (5) and (14), the following differential equation can be obtained:

$$
\begin{aligned}
\dot{p}(t) & +\beta \int_{0}^{t}\left\{-\beta J_{0}[\beta(t-\tau)]+\frac{J_{1}[\beta(t-\tau)]}{t-\tau}\right\} p(\tau) \mathrm{d} \tau \\
= & -\frac{2 \rho c}{m} p(t)-2 \rho c g .
\end{aligned}
$$

Equation (16) gives the governing differential equation for contact force $p(t)$ when the mass moves at subsonic velocity; that is, $V<c$.

Performing the Laplace transformation to (16) gives

$$
\left(\frac{m}{2 \rho c} \frac{s^{2}}{\sqrt{s^{2}+\beta^{2}}}+1\right) \tilde{p}(s)-\frac{m}{2 \rho c} p(0)+\frac{m g}{s}=0,
$$

where $\widetilde{p}(s)$ denotes the Laplace transformation of $p(t)$. Because $p(0)=0$, so

$$
\widetilde{p}(s)=-\frac{2 m g \rho c \sqrt{s^{2}+\beta^{2}}}{s\left(m s^{2}+2 \rho c \sqrt{s^{2}+\beta^{2}}\right)} .
$$

Equation (18) can also be written as

$$
\tilde{p}(s)=m g\left[-\frac{1}{s}+m s \frac{1}{m\left(s^{2}+\beta^{2}\right)+2 \rho c \sqrt{s^{2}+\beta^{2}}-m \beta^{2}}\right] .
$$


Therefore, the inverse Laplace transformation of (19) gives $p(t)=-m g+m^{2} g L^{-1}\left[\frac{s}{m\left(s^{2}+\beta^{2}\right)+2 \rho c \sqrt{s^{2}+\beta^{2}}-m \beta^{2}}\right]$,

where $L^{-1}(\#)$ denotes the inverse Laplace transformation of function \#. According to the following property of Laplace transformation:

$$
L^{-1}[s \tilde{f}(s)]=\frac{\mathrm{d} f(t)}{\mathrm{d} t}, \quad \text { if } f(+0)=0
$$

we have

$$
\begin{aligned}
& L^{-1}\left[s \frac{1}{m\left(s^{2}+\beta^{2}\right)+2 \rho c \sqrt{s^{2}+\beta^{2}}-m \beta^{2}}\right] \\
& \quad=\frac{\mathrm{d}}{\mathrm{d} t} L^{-1}\left[\frac{1}{m\left(s^{2}+\beta^{2}\right)+2 \rho c \sqrt{s^{2}+\beta^{2}}-m \beta^{2}}\right] .
\end{aligned}
$$

Then, by using the following two equations [21, equation (5) in Section 5.1] and [22, equation (9a)]

$$
\begin{aligned}
& L^{-1}\left[\tilde{f}\left(\sqrt{s^{2}+a^{2}}\right)\right]=f(t)-a \int_{0}^{t} f\left(\sqrt{t^{2}-\tau^{2}}\right) J_{1}(a \tau) \mathrm{d} \tau, \\
& L^{-1}\left[\frac{1}{m s^{2}+2 \rho c s-m \beta^{2}}\right] \\
& \quad=\frac{1}{\sqrt{\rho^{2} c^{2}+m^{2} \beta^{2}}} \exp \left(-\frac{\rho c}{m} t\right) \sinh \left(\frac{\sqrt{\rho^{2} c^{2}+m^{2} \beta^{2}}}{m} t\right) .
\end{aligned}
$$

Equation (22) gives

$$
\begin{gathered}
L^{-1}\left[\frac{1}{m\left(s^{2}+\beta^{2}\right)+2 \rho c \sqrt{s^{2}+\beta^{2}}-m \beta^{2}}\right] \\
=\frac{1}{\sigma}\left[\exp \left(-\frac{\rho c t}{m}\right) \sinh \left(\frac{\sigma t}{m}\right)\right. \\
-\beta \int_{0}^{t} \exp \left(-\frac{\rho c}{m} \sqrt{t^{2}-\tau^{2}}\right) \\
\left.\quad \times \sinh \left(\frac{\sigma}{m} \sqrt{t^{2}-\tau^{2}}\right) J_{1}(\beta \tau) \mathrm{d} \tau\right],
\end{gathered}
$$

where

$$
\sigma=\sqrt{\rho^{2} c^{2}+m^{2} \beta^{2}}
$$

Finally, (20), (22), and (24) give

$p(t)$

$$
\begin{aligned}
& \begin{array}{l}
+(\sigma-\rho c) \\
\times \exp \left[\frac{(\sigma-\rho c)}{m} t\right]
\end{array} \\
& \left.-\beta t \int_{0}^{t} \frac{J_{1}(\beta \tau) \exp \left[\frac{(\sigma-\rho c)}{m} \sqrt{t^{2}-\tau^{2}}\right]}{\sqrt{t^{2}-\tau^{2}}} \mathrm{~d} \tau\right\} \\
& =\frac{m g}{2 \sigma}\{+(\sigma+\rho c) \\
& \times\left\{\exp \left[-\frac{(\sigma+\rho c)}{m} t\right]\right. \\
& \left.-\beta t \int_{0}^{t} \frac{J_{1}(\beta \tau) \exp \left[-\frac{(\sigma+\rho c)}{m} \sqrt{t^{2}-\tau^{2}}\right]}{\sqrt{t^{2}-\tau^{2}}} \mathrm{~d} \tau\right\} \\
& -m g \text {. }
\end{aligned}
$$

Equation (25) shows that $\sigma-\rho c>0$ and so the term $\exp [(\sigma-\rho c) t / m]$ in $(26)$ tends to infinity when time tends to infinity, which would cause numerical problem in computation. By using

$$
\exp \left[\frac{(\sigma-\rho c)}{m} t\right]=2 \cosh \left[\frac{(\sigma-\rho c)}{m} t\right]-\exp \left[-\frac{(\sigma-\rho c)}{m} t\right] \text {. }
$$

Equation (26) can be written as $p(t)$

$$
=\frac{m g}{2 \sigma}\left\{\begin{array}{l}
+(\sigma-\rho c) \\
\times\left\{\int_{0}^{t} \frac{J_{1}(\beta \tau) \exp \left[\frac{(\sigma-\rho c)}{m} t\right]-2 \beta t}{+(\sigma+\rho c) \exp \left[-\frac{(\sigma+\rho c)}{m} t\right]}\right. \\
+(\sigma-\rho c) \beta t \\
\quad\left(\int^{t} \frac{J_{1}(\beta \tau) \exp \left[-\frac{(\sigma-\rho c)}{m} \sqrt{t^{2}-\tau^{2}}\right]}{\sqrt{t^{2}-\tau^{2}}} \mathrm{~d} \tau\right. \\
-(\sigma+\rho c) \beta t \\
\times \int_{0}^{t} \frac{J_{1}(\beta \tau) \exp \left[-\frac{(\sigma+\rho c)}{m} \sqrt{t^{2}-\tau^{2}}\right]}{\sqrt{t^{2}-\tau^{2}}} \mathrm{~d} \tau
\end{array}\right.
$$

$-m g$. 
Since [22, equation (3) in Section 6.737 and equation (1) in Section 8.464]

$$
\begin{aligned}
2 t \beta \int_{0}^{t} \frac{J_{1}(\beta \tau) \cosh \left[m^{-1}(\sigma-\rho c) \sqrt{t^{2}-\tau^{2}}\right]}{\sqrt{t^{2}-\tau^{2}}} \mathrm{~d} \tau \\
=t \beta \pi J_{0.5}\left[\frac{t}{2}\left(\sqrt{\beta^{2}-m^{-2}(\sigma-\rho c)^{2}}-i m^{-1}(\sigma-\rho c)\right)\right] \\
\quad \times J_{0.5}\left[\frac{t}{2}\left(\sqrt{\beta^{2}-m^{-2}(\sigma-\rho c)^{2}}+i m^{-1}(\sigma-\rho c)\right)\right] \\
=\exp \left[t m^{-1}(\sigma-\rho c)\right]+\exp \left[-t m^{-1}(\sigma-\rho c)\right] \\
\quad-2 \cos (\omega t),
\end{aligned}
$$

where $i=\sqrt{-1}$ and

$$
\omega=\frac{\sqrt{2 \rho c(\sigma-\rho c)}}{m}
$$

therefore (28) and (29) give

$$
p(t)=m g\left[\frac{\sigma-\rho c}{\sigma} \cos (\omega t)-1\right]+g(t),
$$

where

$$
\begin{aligned}
& g(t) \\
& =\frac{m g}{2 \sigma}\left\{\begin{array}{l}
+(\sigma-\rho c) \beta t \\
\times \int_{0}^{t} \frac{J_{1}(\beta \tau) \exp \left[-m^{-1}(\sigma-\rho c) \sqrt{t^{2}-\tau^{2}}\right]}{\sqrt{t^{2}-\tau^{2}}} \mathrm{~d} \tau \\
-(\sigma+\rho c) \beta t \\
\times \int_{0}^{t} \frac{J_{1}(\beta \tau) \exp \left[-m^{-1}(\sigma+\rho c) \sqrt{t^{2}-\tau^{2}}\right]}{\sqrt{t^{2}-\tau^{2}}} \mathrm{~d} \tau \\
+(\sigma+\rho c) \exp \left(-\frac{\sigma+\rho c}{m} t\right) \\
-(\sigma-\rho c) \exp \left(-\frac{(\sigma-\rho c)}{m} t\right)
\end{array}\right\} .
\end{aligned}
$$

Equation (31) gives the analytical solution for the contact force between the mass and the string when the mass moves at subsonic velocities. Once the contact force is obtained, the displacement of the string can be obtained using (11).

Since the string is infinite, it is worthwhile to see what happens to the contact force when the time tends to infinity. By using variable substitution, the following equation can be obtained as

$$
\begin{aligned}
& \int_{0}^{t} \frac{J_{1}(\beta \tau) \exp \left[-m^{-1}(\sigma-\rho c) \sqrt{t^{2}-\tau^{2}}\right]}{\sqrt{t^{2}-\tau^{2}}} \mathrm{~d} \tau \\
& \quad=\int_{0}^{\pi / 2} J_{1}[\beta t \cos (\theta)] \exp \left[-m^{-1}(\sigma-\rho c) t \sin (\theta)\right] \mathrm{d} \theta .
\end{aligned}
$$

By using $\left|J_{1}(x)\right| \leq 1 / \sqrt{x}, x \geq 0, \beta>0$ and $m^{-1}(\sigma-\rho c)>0$, we have

$$
\begin{aligned}
& \left|\int_{0}^{t} \frac{J_{1}(\beta \tau) \exp \left[-\gamma \sqrt{t^{2}-\tau^{2}}\right]}{\sqrt{t^{2}-\tau^{2}}} \mathrm{~d} \tau\right| \\
& \quad \leq \int_{0}^{\pi / 2} \frac{\exp [-\gamma t \sin (\theta)]}{\sqrt{\beta t \cos (\theta)}} \mathrm{d} \theta=\varphi(t),
\end{aligned}
$$

where

$$
\begin{gathered}
\varphi(t)=\frac{1}{\sqrt{\beta t}}\left[-\frac{4 \gamma t}{5}-\frac{3 \pi^{3 / 2} 2^{1 / 4} \mathbf{L}_{3 / 4}(\gamma t)}{4(\gamma t)^{3 / 4} \Gamma(3 / 4)}\right. \\
\left.+\frac{\pi^{3 / 2}(2 \gamma t)^{1 / 4}}{2 \Gamma(3 / 4)}\left[I_{-1 / 4}(\gamma t)-\mathbf{L}_{7 / 4}(\gamma t)\right]\right], \\
\gamma=m^{-1}(\sigma-\rho c),
\end{gathered}
$$

in which $I_{-1 / 4}(\gamma t)$ denotes the modified Bessel function of the first kind of order $-1 / 4, \mathrm{~L}$ denotes the modified Struve function, and $\Gamma$ denotes the Gamma function. By using Maple software, it is easy to prove that

$$
\lim _{t \rightarrow \infty}[t \varphi(t)]=0 .
$$

Therefore, (33), (34), and (36) give

$$
\lim _{t \rightarrow \infty}\left|t \int_{0}^{t} \frac{J_{1}(\beta \tau) \exp \left[-m^{-1}(\sigma-\rho c) \sqrt{t^{2}-\tau^{2}}\right]}{\sqrt{t^{2}-\tau^{2}}} \mathrm{~d} \tau\right|=0 .
$$

In a similar way, we can prove that

$$
\lim _{t \rightarrow \infty}\left|t \int_{0}^{t} \frac{J_{1}(\beta \tau) \exp \left[-m^{-1}(\sigma+\rho c) \sqrt{t^{2}-\tau^{2}}\right]}{\sqrt{t^{2}-\tau^{2}}} \mathrm{~d} \tau\right|=0 .
$$

By using (32), (37), and (38), we can obtain

$$
\lim _{t \rightarrow \infty} g(t)=0 \text {. }
$$

Then, (31) and (39) give

$$
\lim _{t \rightarrow \infty} p(t)=m g\left[\frac{\sigma-\rho c}{\sigma} \cos (\omega t)-1\right] .
$$

Equation (40) shows that when the mass moves at subsonic velocities and when the time tends to infinity, there exists an asymptotical solution for the contact force.

Furthermore, we consider the asymptotical solution for the displacement of the contact point between the string and the mass. According to (11), when $x=V t$, the displacement of the contact point is given by

$$
u(V t, t)=\frac{1}{2 \rho c} \int_{0}^{t} p(\tau) J_{0}[\beta(t-\tau)] \mathrm{d} \tau .
$$


A combination of (31) and (41) gives

$$
\begin{aligned}
u(V t, t)=\frac{1}{2 \rho c}\left\{\int_{0}^{t}[g(\tau)-m g] J_{0}[\beta(t-\tau)] \mathrm{d} \tau\right. \\
\left.+\frac{m g(\sigma-\rho c)}{\sigma} \int_{0}^{t} \cos [\omega(t-\tau)] J_{0}(\beta \tau) \mathrm{d} \tau\right\} .
\end{aligned}
$$

Firstly, the first term of the right-hand of (42) is considered, which is the convolution of $g(t)$ and $J_{0}(\beta t)$. The limit of the convolution of two functions has the following property [23]; that is, let $f, h[0, \infty) \rightarrow \mathbb{R}$ be locally integrable and assume that

$$
f_{\infty}=\lim _{t \rightarrow \infty} f(t), \quad \int_{0}^{\infty}|g(t)| \mathrm{d} t<\infty,
$$

then

$$
\lim _{t \rightarrow \infty} \int_{0}^{t} h(\tau) f(t-\tau) \mathrm{d} \tau=f_{\infty} \cdot \int_{0}^{\infty} h(\tau) \mathrm{d} \tau .
$$

By using (39) and (44) and the following equation:

$$
\int_{0}^{\infty} J_{0}(\beta \tau) \mathrm{d} \tau=\frac{1}{\beta}
$$

we can obtain

$$
\lim _{t \rightarrow \infty} \int_{0}^{t}[g(\tau)-m g] J_{0}[\beta(t-\tau)] d \tau=\frac{-m g}{\beta} .
$$
as

The second term of the right-hand of (42) can be written

$$
\begin{aligned}
& \int_{0}^{t} \cos [\omega(t-\tau)] J_{0}(\beta \tau) \mathrm{d} \tau \\
& =\cos (\omega t) \int_{0}^{t} \cos (\omega \tau) J_{0}(\beta \tau) \mathrm{d} \tau \\
& \quad+\sin (\omega t) \int_{0}^{t} \sin (\omega \tau) J_{0}(\beta \tau) \mathrm{d} \tau
\end{aligned}
$$

Since $\omega$ can also be written as $\omega=\sqrt{\beta^{2}-m^{-2}(\sigma-\rho c)^{2}}$, so $\beta>\omega>0$, and then the table of integrals gives [22, equations (7) and (8) In Section 6.671]

$$
\begin{gathered}
\lim _{t \rightarrow \infty} \int_{0}^{t} \cos (\omega \tau) J_{0}(\beta \tau) \mathrm{d} \tau=\frac{1}{\sqrt{\beta^{2}-\omega^{2}}}, \\
\lim _{t \rightarrow \infty} \int_{0}^{t} \sin (\omega \tau) J_{0}(\beta \tau) \mathrm{d} \tau=0,
\end{gathered}
$$

and (47) and (48) give

$$
\lim _{t \rightarrow \infty} \int_{0}^{t} \cos [\omega(t-\tau)] J_{0}(\beta \tau) \mathrm{d} \tau=\frac{\cos (\omega t)}{\sqrt{\beta^{2}-\omega^{2}}} .
$$

A combination of (42), (46), and (49) gives

$$
\lim _{t \rightarrow \infty} u(V t, t)=\frac{m g}{2 \rho c}\left[\frac{(\sigma-\rho c)}{\sqrt{\beta^{2}-\omega^{2}}} \cos (\omega t)-\frac{1}{\beta}\right] .
$$

Therefore, when the mass moves at subsonic velocities and when the time tends to infinity, there exists an asymptotical solution for the displacement of the contact point.

\section{The Solutions When the Mass Moves at Supersonic Velocity}

When $V>c$, (12) gives

$$
\frac{\mathrm{d}^{2} u(V t, t)}{\mathrm{d} t^{2}}=0
$$

Then, (5) gives

$$
0=-p(t)-m g
$$

which yields

$$
p(t)=-m g
$$

Therefore, when $V>c,(12)$ and (53) give

$u(x, t)=\frac{-m g}{2 \rho c}\left\{\begin{array}{l}-c t \geq x \\ \int_{0}^{(x+c t) /(c+V)} J_{0}\left[\alpha \sqrt{c^{2}(t-\tau)^{2}-(x-V \tau)^{2}}\right] \mathrm{d} \tau, \\ \int_{(x-c t) /(V-c)}^{(x+c t) /(c+V)} J_{0}\left[\alpha \sqrt{c^{2}(t-\tau)^{2}-(x-V \tau)^{2}}\right] \mathrm{d} \tau, \\ 0, \quad r t \leq x<V t \\ V t \leq x .\end{array}\right.$

Equations (53) and (54) give the analytical contact force and displacement solution of the string, respectively.

Next, the displacement solution for the string at special locations is considered. When $x=V t$, (54) gives

$$
u(V t, t)=0
$$

So, the displacement of the contact point is always zeros when the mass moves at supersonic velocities.

Equations (53) and (55) can be explained as follows. For the case of the supersonic speeds, the velocity of the moving mass is greater than the wave speed, so the string in front of the mass is always still and so the contact force between the mass and the string is $-m g$.

When $x=c t$, (54) gives

$$
u(c t, t)=\frac{-m g}{2 \rho c} \int_{0}^{2 c t /(c+V)} J_{0}\left[\alpha \sqrt{c^{2}(t-\tau)^{2}-(c t-V \tau)^{2}}\right] \mathrm{d} \tau
$$


By using variable substitution, (56) can be rewritten as

$$
u(c t, t)=\frac{-m g t}{2 \rho(c+V)} \int_{0}^{\pi} J_{0}\left[\alpha c t \sqrt{\frac{V-c}{c+V}} \sin (\tau)\right] \sin (\tau) \mathrm{d} \tau .
$$

Then, by using the following equation [22, equation (9) in Section 6.681]:

$$
\int_{0}^{\pi} J_{v}[2 a \sin (y)] \sin (2 \mu y) \mathrm{d} y=\pi \sin (\mu \pi) J_{v-\mu}(a) J_{v+\mu}(a),
$$

we have

$$
u(c t, t)=\frac{-m g}{2 \rho} \frac{\pi t}{c+V} J_{-0.5}\left[\frac{\alpha c t}{2} \sqrt{\frac{V-c}{c+V}}\right] J_{+0.5}\left[\frac{\alpha c t}{2} \sqrt{\frac{V-c}{c+V}}\right] .
$$

Furthermore, using the following equation:

$$
J_{0.5}(z)=\sin (z) \sqrt{\frac{2}{\pi z}}, \quad J_{-0.5}(z)=\cos (z) \sqrt{\frac{2}{\pi z}},
$$

(59) becomes

$$
u(c t, t)=\frac{-m g}{\rho \alpha c \sqrt{V^{2}-c^{2}}} \sin \left(\alpha c t \sqrt{\frac{V-c}{c+V}}\right) .
$$

\section{The Solutions When the Mass Moves at Sonic Velocity}

When $V=c$, (13) gives

$$
\frac{\mathrm{d}^{2} u(V t, t)}{\mathrm{d} t^{2}}=\frac{1}{2 \rho c} \dot{p}(t),
$$

and then (5) gives

$$
\dot{p}(t)=-\frac{2 \rho c}{m} p(t)-2 \rho c g .
$$

The solution of (63) can be given by

$$
p(t)=m g\left[\exp \left(-\frac{2 \rho c}{m} t\right)-1\right] .
$$

Therefore, when the mass moves at sonic velocity, the contact force is given by a very simple formula.

Substituting (64) into (13) and performing some variable substitutions give the following equation:

$$
u(x, t)=\frac{m g}{2 \rho c}\left\{\begin{array}{l}
0, \\
{\left[\frac{x+c t}{2 c} \int_{0}^{1} \exp \left(-\frac{\rho(x+c t)}{m} \tau\right)\right.} \\
\times J_{0}\left[\alpha \sqrt{c^{2} t^{2}-x^{2}} \sqrt{1-\tau}\right] \mathrm{d} \tau \\
\left.-\frac{\sqrt{c^{2} t^{2}-x^{2}} J_{1}\left(\alpha \sqrt{c^{2} t^{2}-x^{2}}\right)}{c \alpha(c t-x)}\right], \\
-c t \leq x \leq c t \\
0, \\
c t<x .
\end{array}\right.
$$

Then, using (65), it is easy to obtain the following two equations:

$$
\begin{aligned}
& \lim _{x \rightarrow c t^{-}} u(x, t)=g\left(\frac{2 \rho c}{m}\right)^{-2}\left[1-\exp \left(-\frac{2 \rho c}{m} t\right)-\frac{2 \rho c}{m} t\right], \\
& \lim _{x \rightarrow c t^{+}} u(x, t)=0 .
\end{aligned}
$$

Therefore, the displacement of the contact point is discontinuous when the mass moves at sonic velocity.

\section{Numerical Results}

In this section, some numerical results for a point mass moving along an infinite string on a Winkler foundation are presented. If we let $\tau=c t$, then (2) becomes

$$
\frac{\partial^{2} u}{\partial \tau^{2}}=\frac{\partial^{2} u}{\partial x^{2}}-\frac{k}{T} u+\frac{1}{T} p(t) \delta\left(x-\frac{V}{c} \tau\right) .
$$

Equation (67) shows that the key parameter is the ratio of the velocity of the mass to the wave speed instead of their absolute values. Therefore, we pay our attention to the different values of the ratio of the velocity of the mass to the wave speed and the following parameters are used. The line mass density and tensile force of the string are $\rho=1(\mathrm{~kg} / \mathrm{m})$ and $T=1(\mathrm{~N})$, respectively; the coefficient of elasticity of the foundation is $k=10\left(\mathrm{~N} / \mathrm{m}^{2}\right)$; the mass of the moving mass is $m=2(\mathrm{~kg})$; and the acceleration due to gravity is $g=10\left(\mathrm{~m} / \mathrm{s}^{2}\right)$. The mass moves in the positive $x$ direction starting from the original point $O$ at a constant speed $V$; see Figure 1 . For the above parameters, the velocity of the wave in the string is $1(\mathrm{~m} / \mathrm{s})$.

To demonstrate the validity of the proposed formula, a comparison with the semianalytical method based on the Fourier analysis is presented. The semianalytical method based on the Fourier analysis [3, Section 3.1.1] reduces the partial differential equation to an ordinary differential equation by applying the Fourier analysis, and the ordinary differential equation is then solved numerically. The accuracy of the semianalytical method depends on both the number of Fourier series and numerical method used to solve the ordinary differential equation. The semianalytical results are obtained for the string with finite length $20(\mathrm{~m})$ and the mass moving at velocity $V=0.4(\mathrm{~m} / \mathrm{s})$ from the middle point of the string to three-quarter length of the sting. In this situation, the semianalytical results are not affected by the string end conditions. The contact force is shown in Figure 3, in which the black, blue, and olive lines correspond to the results obtained by using the semianalytical method with 800,2000 , and 3200 Fourier series, respectively, and the red line corresponds to the results obtained using the proposed formula. Figure 3 shows that as the number of Fourier series increases, the results obtained using the semianalytical method become closer to those obtained from the proposed method.

The contact forces between the mass and the string for $V=0.4,0.6,0.8,1,2$, and $3(\mathrm{~m} / \mathrm{s})$ are given in Figure 4, which shows that the contact force for supersonic velocities 


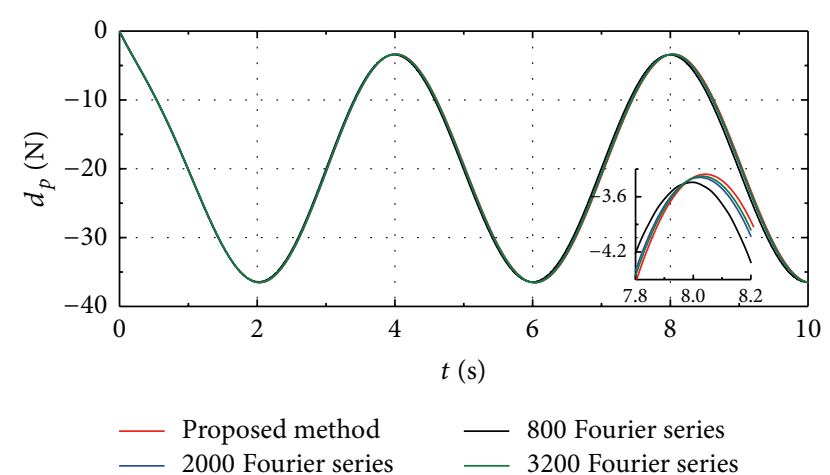

FIGURE 3: The contact force obtained from the proposed formula and the semianalytical method with different numbers of Fourier series when $V=0.4$.

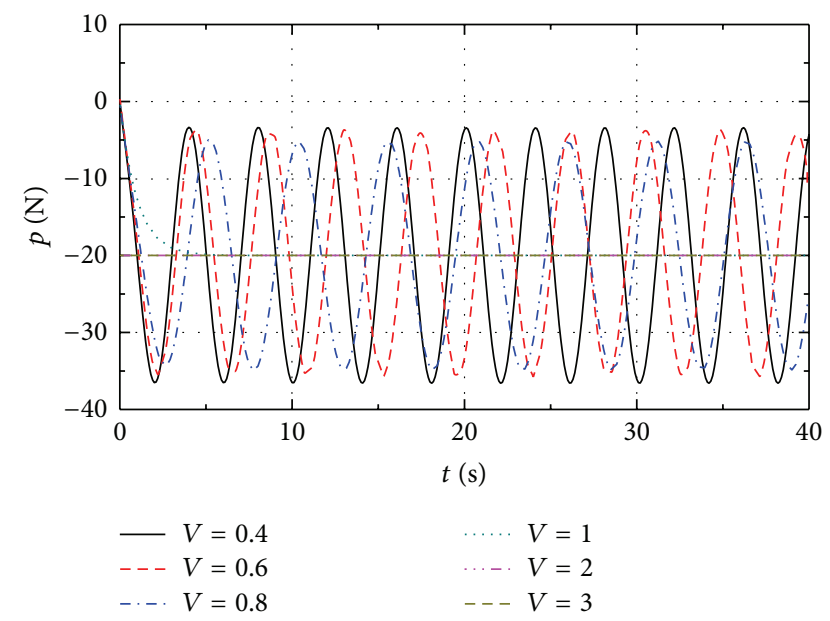

FIGURE 4: The contact force between the mass and the string.

is a constant $m g$ (as shown in Section 4); the contact force for sonic velocity decays exponentially and tends to $m g$, while the contact force for subsonic velocities is oscillating. As shown in Section 3, for subsonic velocities, there exists an asymptotical solution for the contact force when the time tends to infinity. Figure 5 gives the difference $d_{p}$ between the analytical and asymptotical contact forces and shows that the analytical contact force tends to the asymptotical solution when time tends to infinity.

The displacements of the contact point for $V=0.4,0.6$, $0.8,1,2$, and $3(\mathrm{~m} / \mathrm{s})$ are given in Figure 6, which shows that the displacement of the contact point for supersonic velocities is zero, the displacement of the contact point for sonic velocity increases exponentially, while the displacement of the contact point for subsonic velocities is oscillating. As shown in Section 3, for subsonic velocities, the displacement of the contact point has an asymptotical solution when time tends to infinity. Figure 7 gives the difference $d_{u c}$ between the analytical and asymptotical displacements of the contact point and shows that the analytical displacement of the contact point tends to the asymptotical solution when time

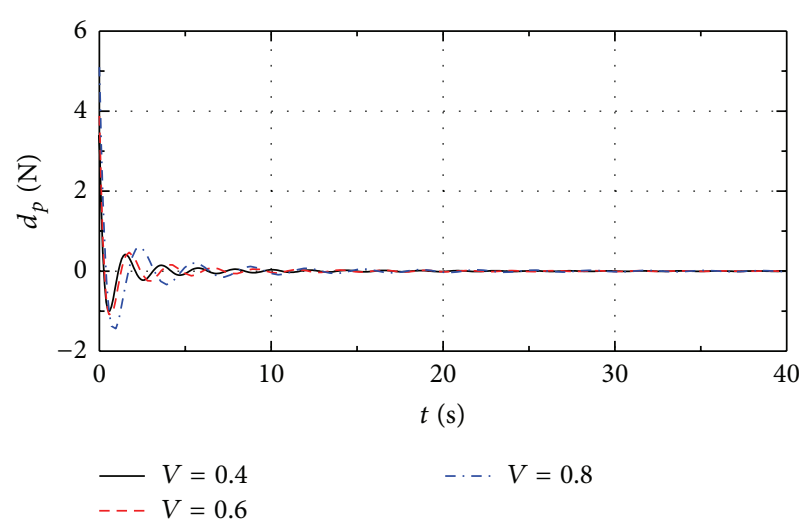

FIGURE 5: The difference between the analytical and asymptotical contact forces.

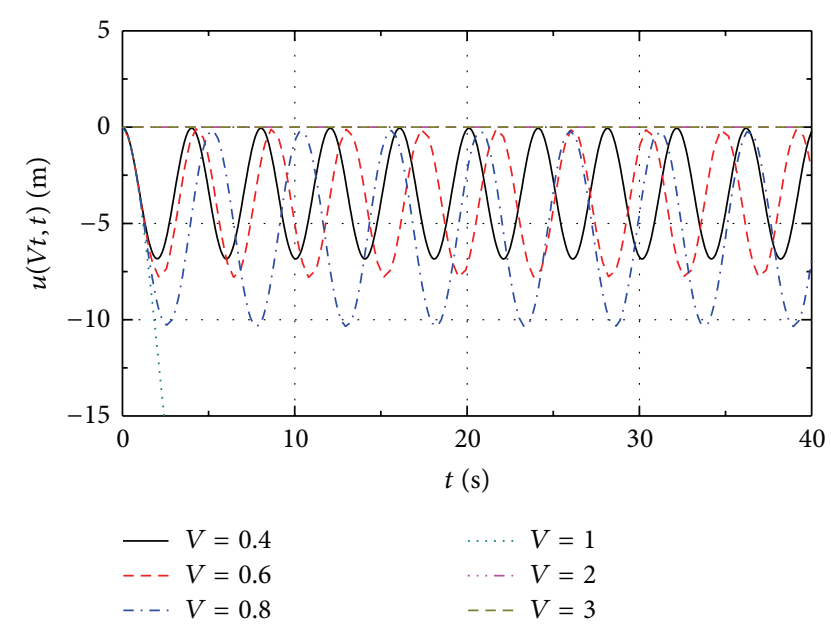

FIgURE 6: The displacement of the contact point.

tends to infinity. For supersonic velocities (i.e., $V=2$ and 3), the displacement of the string at $x=c t$ is given in Figure 8 .

The displacements of the string when the mass moves at subsonic velocities (i.e., $V=0.4,0.6$ and 0.8 ) are given in Figure 9, and the displacements of the string when the mass moves at sonic (i.e., $V=1$ ) and at supersonic velocities (i.e. $V=2$ and 3) are given in Figure 10.

\section{Conclusions}

This paper has presented exact solutions for a point mass moving along a stretched string on a Winkler foundation. The exact solution of the contact force between the string and the mass has been derived, and then the displacement response of the string is given. The solutions cover infinite string subjected to a moving mass at subsonic, sonic, or supersonic velocities. When the mass moves at supersonic velocities, the contact force between the mass and the string is $p(t)=-m g$ and the displacement of the contact point is always zero. When the 


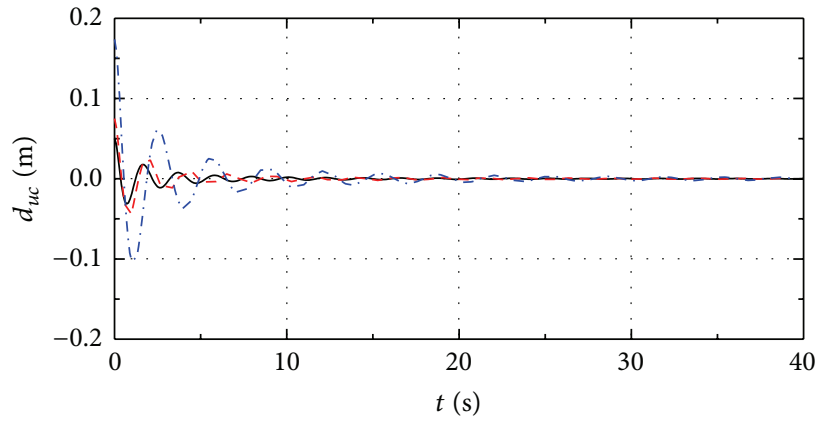

$$
-V=0.4
$$$$
-\cdots \cdot V=0.8
$$

FIGURE 7: The difference between the analytical and asymptotical displacements of the contact point.

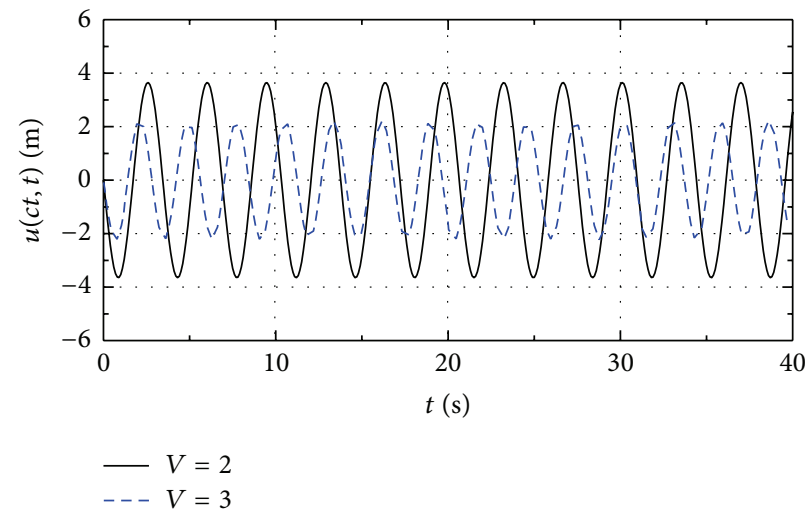

FIGURE 8: The displacement of the string at $x=c t$ when the mass moves at supersonic velocities.

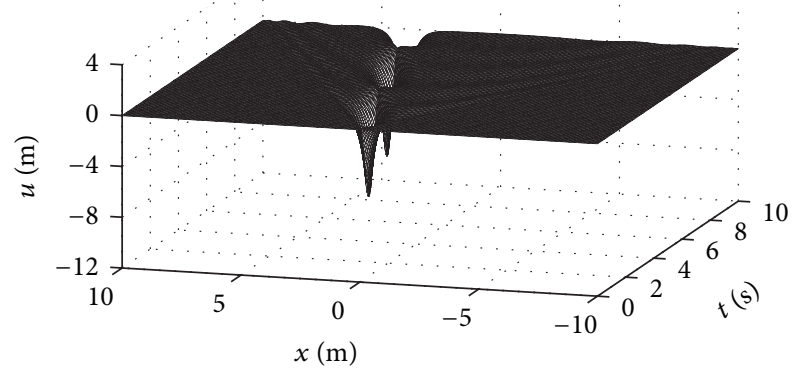

(a)

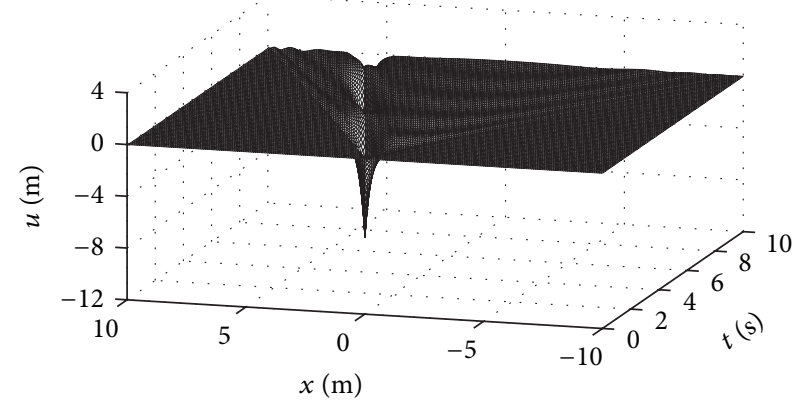

(b)

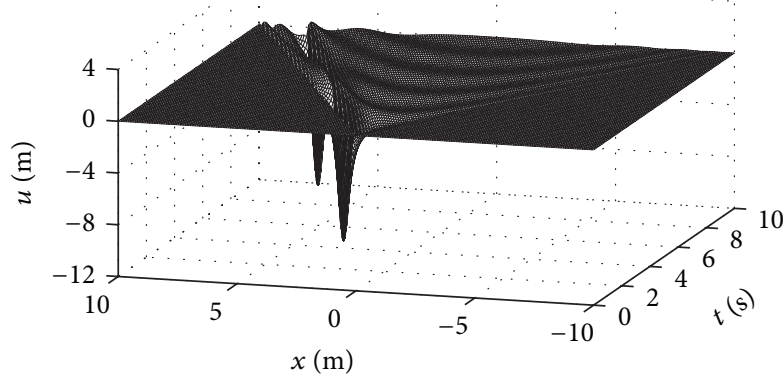

(c)

FIGURE 9: The displacement of the string when the mass moves at subsonic velocities (a) $V=0.4$; (b) $V=0.6$; and (c) $V=0.8$. 


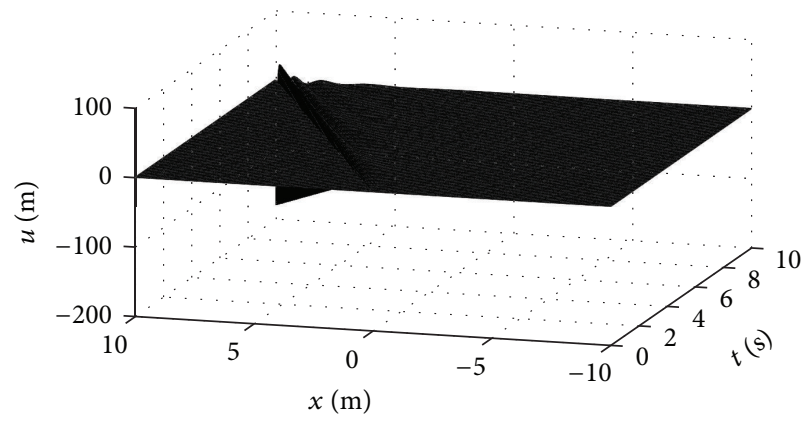

(a)

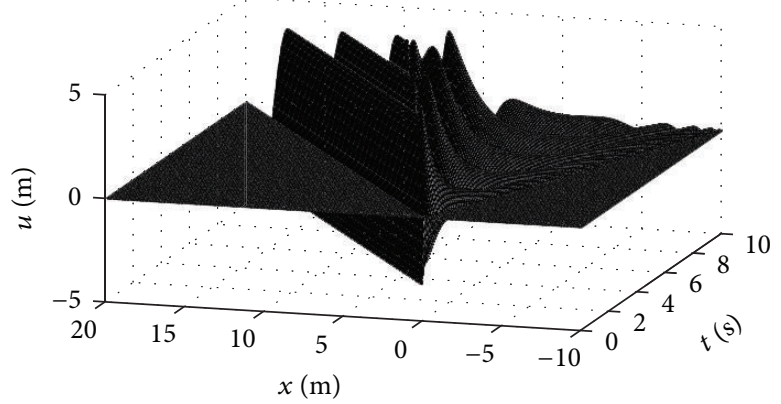

(b)

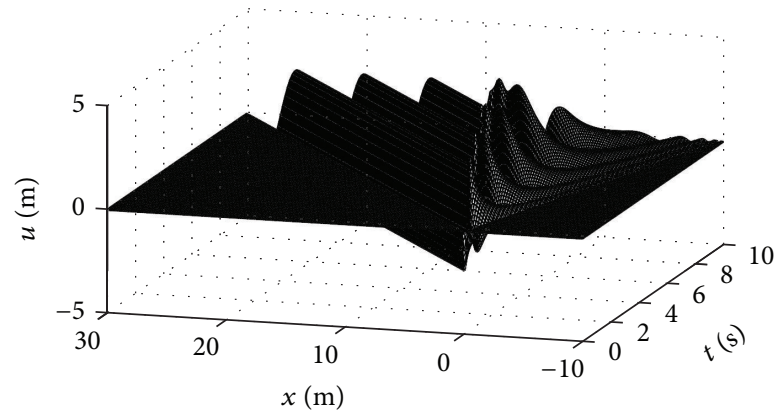

(c)

FIGURE 10: The displacement of the string when the mass moves at sonic or supersonic velocities (a) $V=1$; (b) $V=2$; and (c) $V=3$.

mass moves at sonic velocity, the contact force between the mass and the string is $p(t)=m g[\exp (-2 \rho c t / m)-1]$ and the displacement of the contact point is discontinued. When the mass moves at subsonic velocities, the contact force and the displacement of the contact point are complicated, while their asymptotical solution when time tends to infinity can be given as $\lim _{t \rightarrow \infty} p(t)=m g[((\sigma-$ $\rho c) / \sigma) \cos (\omega t)-1]$ and $\lim _{t \rightarrow \infty} u(V t, t)=(m g / 2 \rho c)[((\sigma-$ $\left.\left.\rho c) / \sqrt{\beta^{2}-\omega^{2}}\right) \cos (\omega t)-1 / \beta\right]$, respectively.

\section{Conflict of Interests}

The authors declare that there is no conflict of interests regarding the publication of this paper.

\section{Acknowledgments}

The authors are grateful for the support of: the Natural Science Foundation of China (no. 11272076); the 973 Program (no. 2011CB711105); Key Laboratory Fund of Liaoning (no. L2013019); the Fundamental Research Funds for the Central Universities (no. DUT13LK12 and no. DUT14YQ202); and Program for New Century Excellent Talents in University (NCET-13-0072).

\section{References}

[1] L. Frýba, Vibration of Solids and Structures Under Moving Loads, Thomas Telford, London, UK, 3rd edition, 1999.
[2] H. Ouyang, "Moving-load dynamic problems: a tutorial (with a brief overview)," Mechanical Systems and Signal Processing, vol. 25, no. 6, pp. 2039-2060, 2011.

[3] C. I. Bajer and B. Dyniewicz, Numerical Analysis of Vibrations of Structures Under Moving Inertial Load, Springer, Berlin, Germany, 2012.

[4] M. F. Kanninen and A. L. Florence, "Traveling forces on strings and membranes," International Journal of Solids and Structures, vol. 3, no. 2, pp. 143-154, 1967.

[5] F. T. Flaherty Jr., "Transient resonance of an ideal string under a load moving with varying speed," International Journal of Solids and Structures, vol. 4, no. 12, pp. 1221-1231, 1968.

[6] M. J. Sagartz and M. J. Forrestal, "Motion of a stretched string loaded by an accelerating force," Journal of Applied Mechanics, vol. 42, no. 2, pp. 505-506, 1975.

[7] A. R. M. Wolfert, H. A. Dieterman, and A. V. Metrikine, "Passing through the "elastic wave barrier" by a load moving along a waveguide," Journal of Sound and Vibration, vol. 203, no. 4, pp. 597-606, 1997.

[8] S. Gavrilov, "Non-stationary problems in dynamics of a string on an elastic foundation subjected to a moving load," Journal of Sound and Vibration, vol. 222, no. 3, pp. 345-361, 1999.

[9] H. A. Dieterman and A. V. Kononov, "Uniform motion of a constant load along a string on an elastically supported membrane," Journal of Sound and Vibration, vol. 208, no. 4, pp. 575$586,1997$.

[10] A. V. Kononov and H. A. Dieterman, "The elastic field generated by two loads moving along two strings on an elastically supported membrane," Journal of Sound and Vibration, vol. 214, no. 4, pp. 725-746, 1998. 
[11] J. Rusin, P. Śniady, and P. Śniady, "Vibrations of double-string complex system under moving forces. Closed solutions," Journal of Sound and Vibration, vol. 330, no. 3, pp. 404-415, 2011.

[12] C. E. Smith, "Motions of a stretched string carrying a moving mass particle," Journal of Applied Mechanics, vol. 31, no. 1, pp. 29-37, 1964.

[13] R. Rodeman, D. B. Longcope, and L. F. Shampine, "Response of a string to an accelerating mass," Journal of Applied Mechanics, vol. 43, no. 4, pp. 675-680, 1976.

[14] B. Yang, C. A. Tan, and L. A. Bergman, "Direct numerical procedure for solution of moving oscillator problems," Journal of Engineering Mechanics, vol. 126, no. 5, pp. 462-469, 2000.

[15] C. I. Bajer and B. Dyniewicz, "Space-time approach to numerical analysis of a string with a moving mass," International Journal for Numerical Methods in Engineering, vol. 76, no. 10, pp. 1528-1543, 2008.

[16] B. Dyniewicz and C. I. Bajer, "Paradox of a particle's trajectory moving on a string," Archive of Applied Mechanics, vol. 79, no. 3, pp. 213-223, 2009.

[17] H. Kruse, K. Popp, and A. V. Metrikine, "Eigenfrequencies of a two-mass oscillator uniformly moving along a string on a viscoelastic foundation," Journal of Sound and Vibration, vol. 218, no. 1, pp. 103-116, 1998.

[18] K. Lee, Y. Cho, and J. Chung, "Dynamic contact analysis of a tensioned beam with a moving massspring system," Journal of Sound and Vibration, vol. 331, no. 11, pp. 2520-2531, 2012.

[19] S. N. Gavrilov and D. A. Indeitsev, "The evolution of a trapped mode of oscillations a "string on an elastic foundation-moving inertial inclusion" system," Journal of Applied Mathematics and Mechanics, vol. 66, no. 5, pp. 825-833, 2002.

[20] E. Zauderer, Partial Differential Equations of Applied Mathematics, John Wiley \& Sons, New York, NY, USA, 2nd edition, 1989.

[21] H. Bateman, Tables of Integral Transforms, vol. 1, McGraw Hill, New York, NY, USA, 1954.

[22] I. S. Gradshteyn and I. M. Ryzhik, Table of Integrals, Series and Products, Academic Press, Boston, Mass, USA, 7th edition, 2007.

[23] I. Gyori and L. Horváth, "New limit formulas for the convolution of a function with a measure and their applications," Journal of Inequalities and Applications, vol. 2008, Article ID 748929, 35 pages, 2008. 

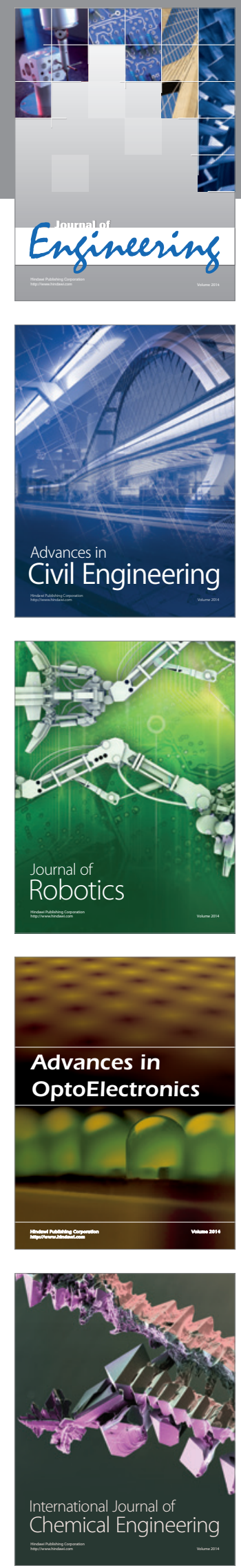

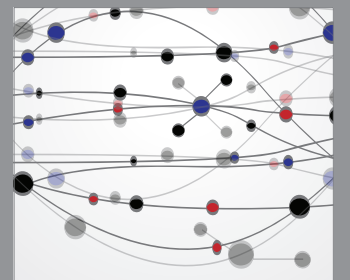

The Scientific World Journal
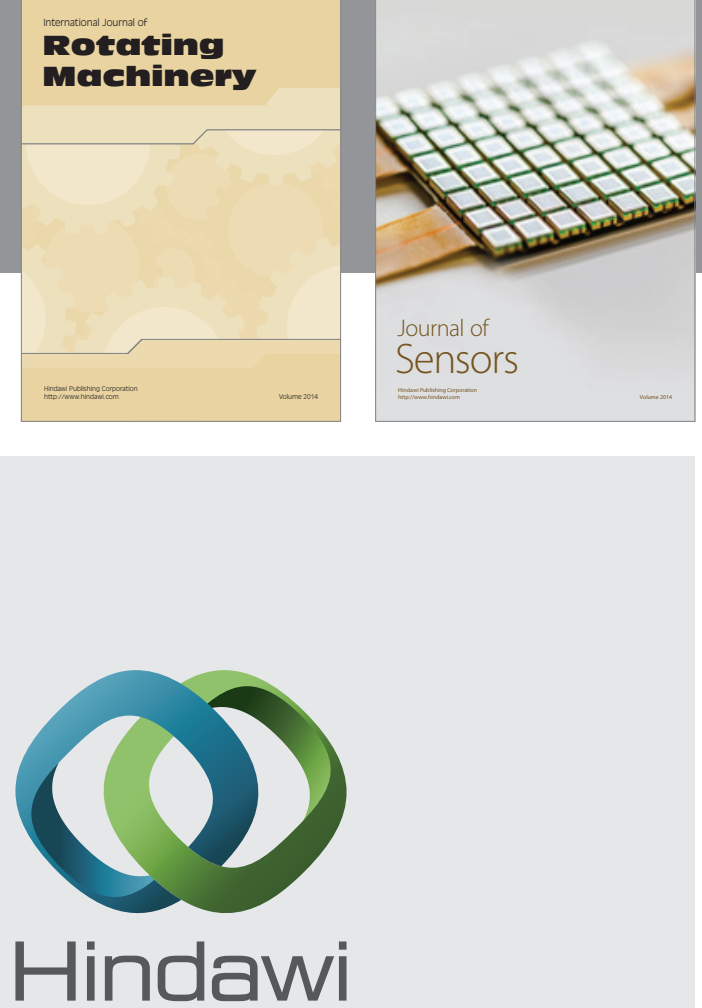

Submit your manuscripts at http://www.hindawi.com
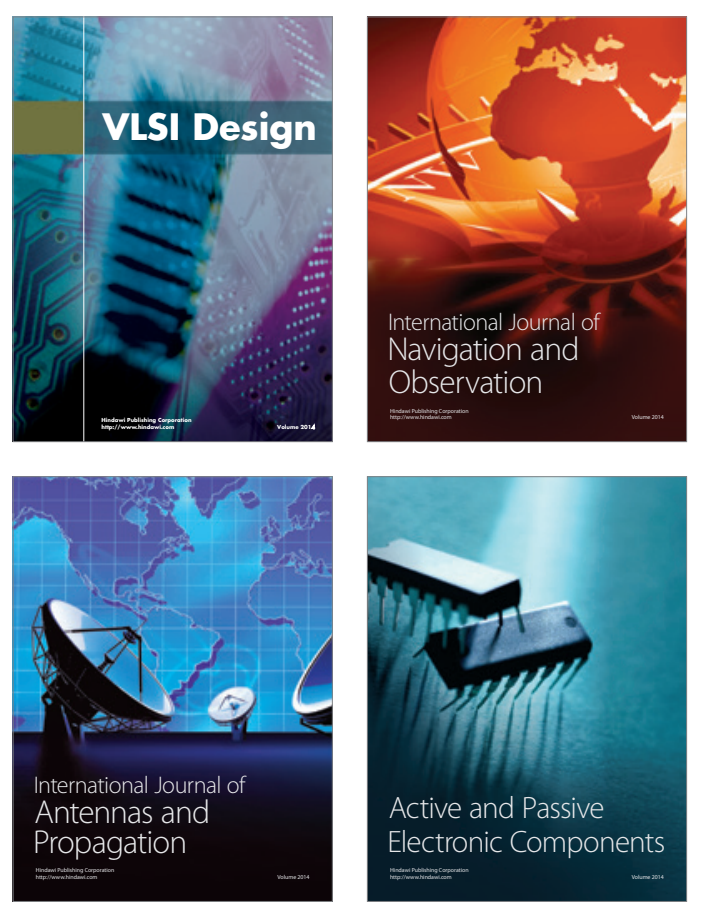
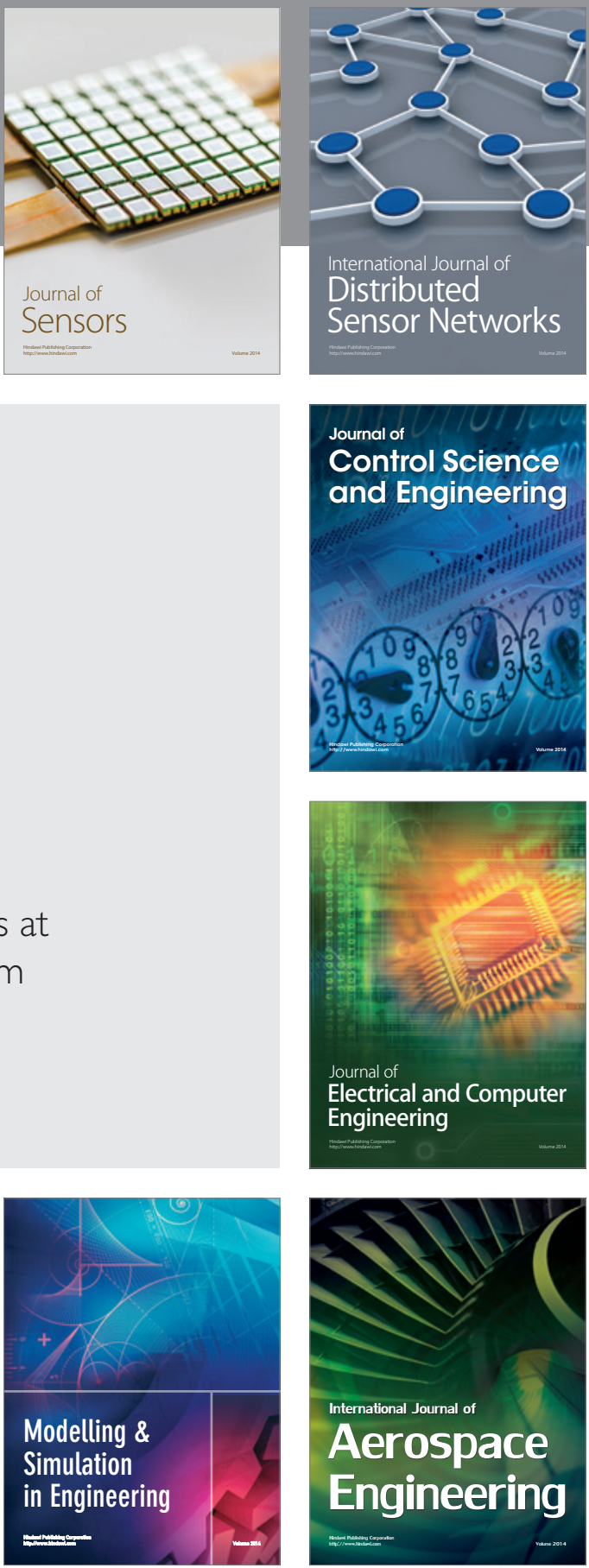

Journal of

Control Science

and Engineering
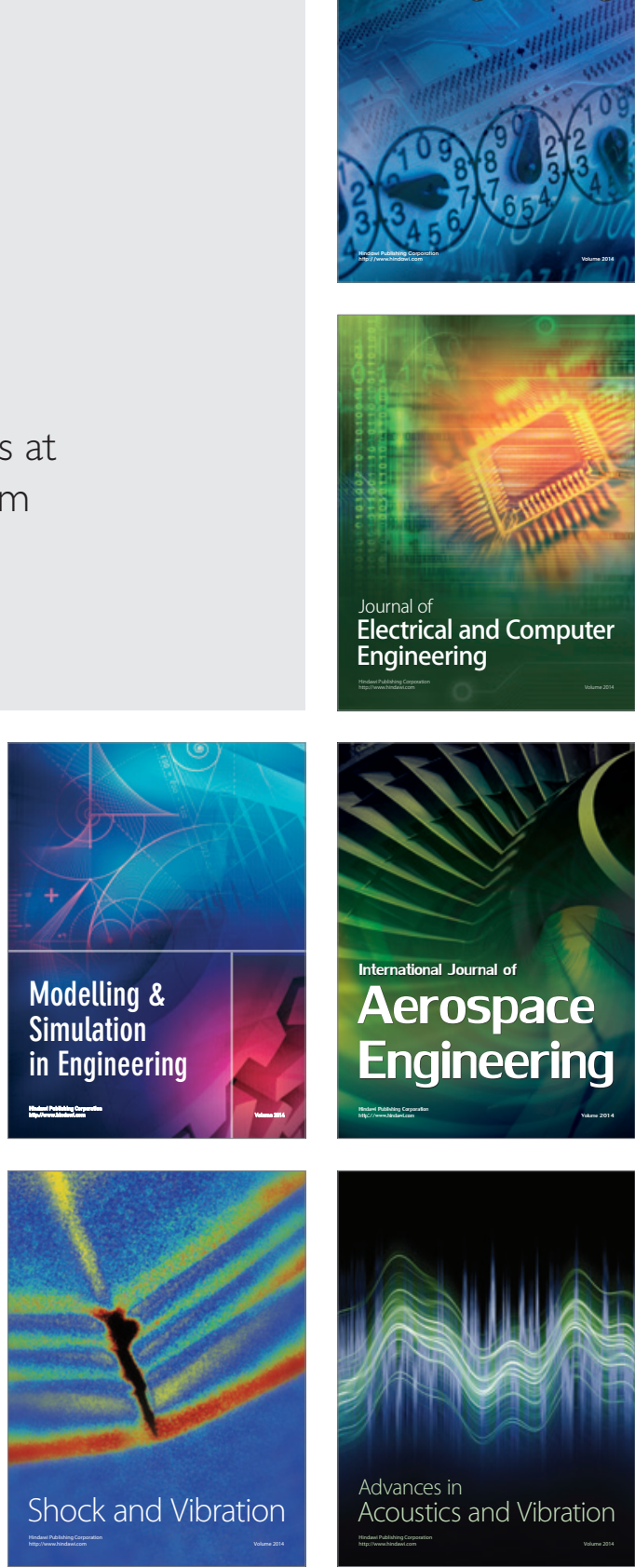